\title{
Adrenal venous sampling in patients with ACTH-independent hypercortisolism
}

\author{
Eleni Papakokkinou $\mathbb{1}^{1,2} \cdot$ Hugo Jakobsson ${ }^{1,2} \cdot$ Augustinas Sakinis $^{3} \cdot$ Andreas Muth $^{4} \cdot$ Bo Wängberg $^{4} \cdot$ Olof Ehn $^{1,2}$. \\ Gudmundur Johannsson ${ }^{1,2} \cdot$ Oskar Ragnarsson ${ }^{1,2}$
}

Received: 15 May 2019 / Accepted: 26 July 2019 / Published online: 22 August 2019

(c) The Author(s) 2019

\begin{abstract}
Purpose To study the usefulness of adrenal venous sampling (AVS) in distinguishing unilateral from bilateral cortisol production in patients with $\mathrm{ACTH}$-independent hypercortisolism and bilateral adrenal lesions, or morphologically normal adrenal glands.

Methods A retrospective analysis of ten consecutive patients with ACTH-independent hypercortisolism who underwent AVS at our institution between 2009 and 2017. Unilateral dominant cortisol production was defined as a side-to-side cortisol/aldosterone lateralization ratio $>2$.

Results Four of ten patients had overt Cushing's syndrome. Of these, two had bilateral adrenal lesions on computed tomography and two had normal adrenal glands. One of the two patients with bilateral adrenal lesions had, based on the AVS, a unilateral dominant cortisol production. Following unilateral adrenalectomy the patient developed adrenal insufficiency. The other three patients were considered to have bilateral cortisol production and underwent bilateral adrenalectomy. Six patients had a mild autonomous cortisol secretion and bilateral adrenal lesions. Based on AVS, one patient was considered to have unilateral dominant cortisol production, underwent unilateral adrenalectomy and developed transient adrenal insufficiency postoperatively.

Conclusions AVS may contribute to appropriate treatment in patients with ACTH-independent hypercortisolism and bilateral adrenal lesions. In our series, AVS was helpful in the decision-making of two out of ten patients, avoiding chronic treatment with steroidogenesis inhibitors, or inappropriate bilateral adrenalectomy.
\end{abstract}

Keywords Cushing's syndrome $\cdot$ Adrenal venous sampling $\cdot$ Hypercortisolism $\cdot$ ACTH-independent $\cdot$ Autonomous cortisol secretion

\section{Abbreviations \\ ACTH adrenocorticotropic hormone \\ CS Cushing's syndrome \\ MACS mild autonomous cortisol secretion}

Eleni Papakokkinou

eleni.papakokkinou@vgregion.se

1 Department of Internal Medicine and Clinical Nutrition, Institute of Medicine at Sahlgrenska Academy, University of Gothenburg, Gothenburg, Sweden

2 Department of Endocrinology, Sahlgrenska University Hospital, Gothenburg, Sweden

3 Department of Radiology, Sahlgrenska University Hospital, Gothenburg, Sweden

4 Department of Surgery, Institute of Clinical Sciences, Sahlgrenska Academy, University of Gothenburg, Sahlgrenska University Hospital, Gothenburg, Sweden
AVS adrenal venous sampling

PBMAH primary bilateral macronodular hyperplasia

PPNAD primary pigmented nodular adrenocortical disease

LDDST low-dose dexamethasone suppression test

DHEAS dehydroepiandrosterone sulfate

BMI body mass index

CT computed tomography

\section{Introduction}

Adrenal Cushing's syndrome (CS) is characterized by autonomous cortisol production with low plasma ACTH concentrations and is therefore defined as ACTHindependent CS. Overall, ACTH-independent CS accounts for $15-20 \%$ of endogenous CS where the most common cause is a unilateral cortisol producing adrenal adenoma [1]. 
Other rarer causes of ACTH-independent CS are primary bilateral macronodular adrenal hyperplasia (PBMAH) and primary pigmented nodular adrenocortical disease (PPNAD) $(<2 \%)$ [2]. Approximately $10 \%$ of patients with ACTH-independent CS have bilateral adrenal lesions [3].

Mild autonomous cortisol secretion (MACS) is defined as a mild hypersecretion of cortisol in patients with adrenal incidentaloma but without the typical signs and symptoms of clinically overt hypercortisolism. MACS is present in $5-30 \%$ of patients with incidentally discovered adrenal adenomas [4]. Importantly, 9-17\% of patients with adrenal incidentalomas have bilateral lesions [5, 6], and seem to have an even higher prevalence of MACS than patients with unilateral lesions [7].

While diagnostic work-up and treatment of patients with ACTH-independent CS and unilateral adenomas is usually straightforward, management of patients with bilateral adrenal lesions is more challenging since distinction between a functioning and nonfunctioning adrenal lesion cannot be reliably determined by specific radiological or clinical features [8]. The same applies to patients with ACTH-independent CS and morphologically normal adrenal glands.

Adrenal venous sampling (AVS) is an established method to distinguish unilateral from bilateral aldosterone overproduction [9]. The usefulness of AVS for differentiating unilateral from bilateral cortisol overproduction in patients with ACTH-independent hypercortisolism has however only been evaluated in two studies. Young et al. found AVS to be helpful for deciding treatment strategy in ten patients with ACTH-independent CS and bilateral adrenal masses [10] and, recently, Ueland et al. found that four of fourteen patients with MACS and bilateral adrenal lesions had unilateral cortisol hypersecretion [8]. Otherwise, the diagnostic value of AVS in this context is limited to case reports [11-19].

Hereby we report our experience using AVS in ten patients with ACTH-independent hypercortisolism.

\section{Material and methods}

\section{Patients}

This was a retrospective analysis of ten consecutive patients with ACTH-independent hypercortisolism that were subjected to AVS at our institution between 2009 and 2017. Eight patients had been referred for evaluation of incidentally found bilateral adrenal lesions and two due to clinically overt CS. All patients were initially evaluated clinically and biochemically (Table 1). In all patients, ACTH was measured at $8 \mathrm{a} . \mathrm{m}$. and serum cortisol at midnight, an overnight $1 \mathrm{mg}$ dexamethasone suppression test was performed, and
$24 \mathrm{~h}$ urine was sampled for measurement of urinary free cortisol (UFC). All patients were evaluated with low-dose dexamethasone suppression test (LDDST; $0.5 \mathrm{mg}$ every sixth hour for 2 days). MACS was defined as absence of typical clinical signs and symptoms of CS, pathological dexamethasone suppression test $(>50 \mathrm{nmol} / \mathrm{L})$ and normal UFC in patients with adrenal incidentaloma. In addition, at least two of the following needed to be fulfilled: (a) low or low normal ACTH concentrations in plasma $(<4.0 \mathrm{pmol} / \mathrm{L})$, (b) serum cortisol at midnight $>100 \mathrm{nmol} / \mathrm{L}$, and (c) serum cortisol $>50 \mathrm{nmol} / \mathrm{L}$ following LDDST.

In addition, one patient with suspected ACTHindependent Cushing's syndrome, due to low to normal ACTH levels and a suspected $9 \mathrm{~mm}$ right-sided adrenal adenoma, underwent AVS during the study period. Eventually, this patient was found to have cyclic Cushing's disease and was successfully treated with transsphenoidal pituitary adenomectomy. For illustrative purposes, the data for this patient is presented in Tables 1-3 but is otherwise excluded from the analyses.

\section{Adrenal venous sampling}

AVS was performed on the second day after 48-h dexamethasone suppression ( $0.5 \mathrm{mg}$ every sixth hour). The AVS procedure was performed as previously described [20]. In short, the right femoral vein was punctured and a SIM 1 catheter, Cobra, or Shepherd Hook was used to localize and draw blood from the right adrenal vein. Next, the left adrenal vein was localized and sampled by using a SIM 2 or SIM 3 catheter. Finally, blood samples from the inferior vena cava and a peripheral vein were drawn.

Concentrations of serum cortisol $(n=10)$, aldosterone $(n=10)$, DHEAS $(n=9)$, plasma adrenaline $(n=8)$, and noradrenaline $(n=8)$ were measured. The adequacy of the cannulation was based on the adrenal to peripheral vein ratio of aldosterone of $>2$, in accordance with the selectivity index used during AVS in patients with primary aldosteronism (without cosyntropin use) [9, 21]. The lateralization of the cortisol secretion was determined by calculation of the side-to-side adrenal vein cortisol concentration to aldosterone, adrenaline, noradrenaline, and DHEAS, respectively [(Cortisol/Reference hormone Dominant adrenal vein $) /$ (Cortisol/Reference hormone $\left.{ }_{\text {Non-dominant adrenal vein }}\right)$. Since no consensus exists on how to optimally define laterality during AVS in patients with CS, each patient was evaluated individually. However, in general, a patient was considered to have a unilateral dominant cortisol production when the side-to-side lateralization ratio was $>2$, when using aldosterone as a reference hormone, especially in conjunction with a concordant side-to-side lateralization ratio $>2$ using the other three hormones as reference. When the concentration of the aldosterone, adrenaline, or noradrenaline 


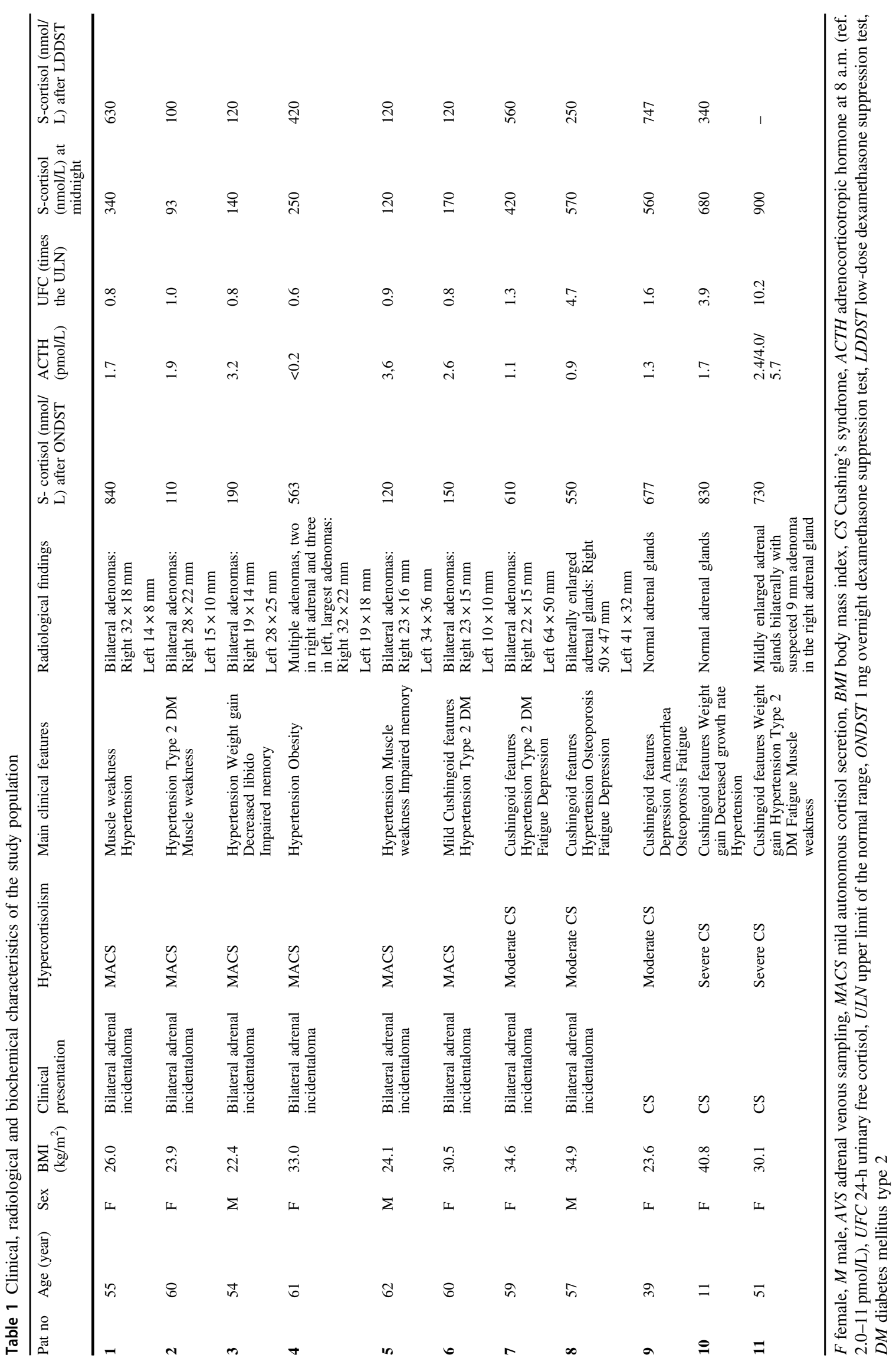


was unmeasurably low, the lower limit of detection was used for calculation of selectivity and lateralization.

The decision for the treatment in all cases was based on the severity of hypercortisolism, laboratory data, imaging findings, as well as findings from the AVS and the patient's preference.

\section{Assays}

Serum cortisol was measured by using competitive electrochemiluminescence immunoassays; Roche Modular E (2009-2011; CV 5-7\%), Roche Cobas, Cortisol 1 (2011-2015; CV 3-4\%), and Roche Cobas, Cortisol-II (2015-2017; CV 2-3 \%). Serum aldosterone was measured with radioimmunoassays; Siemens Coat-A-Count (November 2009-October 2014; CV 6-10\%) and DiaSorin Liaison (November 2014-2017; CV 8-13\%). Serum DHEAS was measured with a radioimmunoassay Perkin- Elmer Coat-ACount, DPC Scandinavia AB (2009-2010; CV 12\%), and with an immunoenzymatic method Beckman Coulter (2010-2017; CV 10\%). Plasma ACTH was measured with an ELSA immunoradiometric assay (2009-2016; CV 6.8-14\%) and with an electrochemiluminescence immunoassay for adrenocorticotropic hormone on a Cobas E Analyzer (2016-2017; CV 3.04-3.8\%). Plasma adrenaline and noradrenaline were measured with high performance liquid chromatography with electrochemical detection (Chromeleon computer system; 2009-2017; CV for adrenaline $7-8 \%$ and $6 \%$ for noradrenaline).

\section{Statistical methods}

The statistical analyses were performed with SPSS, version 22.0 for Windows. Continuous variables are presented as median (25-75 percentiles; range) and categorical variables as number $(\%)$.

\section{Results}

\section{Baseline characteristics}

Eleven patients, eight women and three men, were studied with AVS between 2009 and 2017. The median (25-75 percentiles; range) age at diagnosis was 57 (51-61; 11-62) years (Table 1).

Eight patients were initially referred for evaluation of adrenal incidentaloma, six of these had MACS and two had clinically overt CS (Table 1). Two patients were referred due to overt ACTH-independent CS. Thus, in total, four patients had clinically overt CS. Two of these had bilateral adrenal lesions and two had normal adrenal glands. All patients with MACS had bilateral adrenal lesions.
All patients were considered to be successfully catheterized. Nine of ten patients had adrenal to peripheral vein ratio of aldosterone $>2$ (selectivity index). The mean selectivity index was 31 (range 4.0-98) and 24 (range 5.9-65) for the left and right adrenal veins, respectively (Table 2). In addition, the AVS in one patient (subject 10), with selectivity index $<2$, was considered to be successful based on more than ten times higher concentrations of cortisol, and adrenaline, from the adrenal veins compared to peripheral vein.

\section{Patients with MACS}

Four women and two men had bilateral adrenal incidentalomas and MACS (Subjects 1-6; Tables 1-3). All had normal UFC and none had suppressed serum cortisol following overnight low-dose dexamethasone test. One patient (Subject 1; Fig. 1) was considered to have dominant cortisol production from the right adrenal gland based on a right to left cortisol/aldosterone gradient of 2.6 (Subject 1; Tables 1-3). Right to left cortisol/noradrenaline, cortisol/adrenaline and cortisol/DHEAS gradients were 3.6, 4.2, and 1.5, respectively (Table 2). The patient underwent unilateral adrenalectomy and developed adrenal insufficiency postoperatively. Histopathological examination showed a benign adrenal adenoma.

Based on the side-to-side gradients, four patients were considered to have bilateral MACS. In addition, one patient (Subject 6; Table 2) with concomitant primary aldosteronism was considered to have bilateral MACS based on relatively low side-to-side cortisol/noradrenaline, cortisol/ adrenaline, and cortisol/DHEAS gradients. None of these five patients had overt CS and received therefore either medical treatment or active surveillance (Tables 2 and 3).

\section{Patients with overt hypercortisolism}

Two patients had overt hypercortisolism and bilateral adrenal lesions (Subjects 7 and 8; Figs. 2 and 3). One of them had unilateral dominant cortisol production based on a left-to-right cortisol/aldosterone gradient of 7.1 and underwent left-sided adrenalectomy (Subject 7; Tables 1-3). The histopathological diagnosis was benign adrenal adenoma. Postoperatively, the patient developed adrenal insufficiency and all clinical features of hypercortisolism resolved. The other patient (Subject 8; Tables 1-3) had bilateral cortisol overproduction and was treated with bilateral adrenalectomy. The histopathological diagnosis was PBMAH.

Two patients (Subjects 9 and 10; Tables 1-3) had overt hypercortisolism and morphologically normal adrenal glands on CT. Both had bilateral cortisol overproduction and were treated with bilateral adrenalectomy. Histopathological examination confirmed PPNAD in both cases. 
Table 2 Results from adrenal venous sampling in 11 patients with hypercortisolism

\begin{tabular}{|c|c|c|c|c|c|c|c|c|}
\hline & Peripheral & Vena cava & Left adrenal & Right adrenal & L SI & R SI & $\mathrm{R} / \mathrm{L}$ gradient & $\mathrm{L} / \mathrm{R}$ gradient \\
\hline \multicolumn{9}{|l|}{ Patient 1} \\
\hline Cortisol (nmol/L) & 480 & 480 & 2400 & 6040 & & & & \\
\hline Aldosterone (pmol/L) & 54 & 79 & 564 & 539 & 10.4 & 10.0 & 2.6 & \\
\hline Noradrenaline $(\mathrm{nmol} / \mathrm{L})$ & - & 0.8 & 22 & 16 & & & 3.6 & \\
\hline Adrenaline (nmol/L) & - & 0.2 & 97 & 58 & & & 4.2 & \\
\hline DHEA-s $(\mu \mathrm{mol} / \mathrm{L})$ & 1.7 & 2.2 & 2.2 & 3.8 & & & 1.5 & \\
\hline \multicolumn{9}{|l|}{ Patient 2} \\
\hline Cortisol (nmol/L) & 140 & 140 & 520 & 940 & & & & \\
\hline Aldosterone (pmol/L) & 52 & 60 & 228 & 307 & 4.4 & 5.9 & 1.3 & \\
\hline Noradrenaline $(\mathrm{nmol} / \mathrm{L})$ & - & - & - & - & & & - & \\
\hline Adrenaline $(\mathrm{nmol} / \mathrm{L})$ & - & - & - & - & & & - & \\
\hline DHEA-s $(\mu \mathrm{mol} / \mathrm{L})$ & 0.76 & 0.69 & 0.9 & 1.0 & & & 1.6 & \\
\hline \multicolumn{9}{|l|}{ Patient 3} \\
\hline Cortisol (nmol/L) & 210 & 220 & 1710 & 1520 & & & & \\
\hline Aldosterone (pmol/L) & 78 & 90 & 1830 & 2260 & 23.5 & 29.0 & & 1.4 \\
\hline Noradrenaline $(\mathrm{nmol} / \mathrm{L})$ & 1.3 & 1.0 & 1.7 & 15 & & & & 10 \\
\hline Adrenaline (nmol/L) & 0.1 & 0.1 & 4.2 & 57 & & & & 14 \\
\hline DHEA-s $(\mu \mathrm{mol} / \mathrm{L})$ & 0.6 & 0.6 & 0.6 & 0.8 & & & & 1.3 \\
\hline \multicolumn{9}{|l|}{ Patient 4} \\
\hline Cortisol (nmol/L) & 330 & 300 & 1650 & 2670 & & & & \\
\hline Aldosterone (pmol/L) & 141 & 208 & 559 & 1790 & 4.0 & 12.7 & & 2.0 \\
\hline Noradrenaline $(\mathrm{nmol} / \mathrm{L})$ & - & - & - & - & & & - & \\
\hline Adrenaline $(\mathrm{nmol} / \mathrm{L})$ & - & - & - & - & & & - & \\
\hline DHEA-s $(\mu \mathrm{mol} / \mathrm{L})$ & - & - & - & - & & & - & \\
\hline \multicolumn{9}{|l|}{ Patient 5} \\
\hline Cortisol (nmol/L) & 120 & - & 1320 & 1830 & & & & \\
\hline Aldosterone (pmol/L) & $<30$ & - & 622 & 1960 & 20.7 & 65.3 & & 2.3 \\
\hline Noradrenaline $(\mathrm{nmol} / \mathrm{L})$ & 0.5 & 0.9 & 2.4 & 1.8 & & & 1.8 & \\
\hline Adrenaline (nmol/L) & 0.3 & 0.1 & 7.0 & 159 & & & & 16 \\
\hline DHEA-s $(\mu \mathrm{mol} / \mathrm{L})$ & 1.6 & 1.6 & 1.9 & 1.6 & & & 1.6 & \\
\hline \multicolumn{9}{|l|}{ Patient 6} \\
\hline Cortisol (nmol/L) & 100 & 110 & 790 & 1450 & & & & \\
\hline Aldosterone (pmol/L) & 321 & 279 & 31500 & 5540 & 98.1 & 17.3 & 10.4 & \\
\hline Noradrenaline $(\mathrm{nmol} / \mathrm{L})$ & 0.5 & 0.9 & 2.4 & 1.8 & & & 2.5 & \\
\hline Adrenaline (nmol/L) & $<0.02$ & $<0.02$ & 6.8 & 6.3 & & & 2.0 & \\
\hline DHEA-s $(\mu \mathrm{mol} / \mathrm{L})$ & 0.6 & 0.6 & 0.9 & 0.9 & & & 1.8 & \\
\hline \multicolumn{9}{|l|}{ Patient 7} \\
\hline Cortisol (nmol/L) & 540 & 570 & 1620 & 980 & & & & \\
\hline Aldosterone (pmol/L) & 57 & 96 & 318 & 1340 & 5.6 & 23.5 & & 7.1 \\
\hline Noradrenaline $(\mathrm{nmol} / \mathrm{L})$ & 1.9 & 2.2 & 1.1 & 8.4 & & & & 12.5 \\
\hline Adrenaline (nmol/L) & 0.3 & $<0.16$ & 0.2 & 40 & & & & 330 \\
\hline DHEA-s $(\mu \mathrm{mol} / \mathrm{L})$ & 7 & 7 & 7.7 & 7.4 & & & & 1.6 \\
\hline \multicolumn{9}{|l|}{ Patient 8} \\
\hline Cortisol (nmol/L) & 390 & 390 & 1000 & 1530 & & & & \\
\hline Aldosterone (pmol/L) & 46 & 50 & 268 & 375 & 5.8 & 8.1 & 1.1 & \\
\hline Noradrenaline $(\mathrm{nmol} / \mathrm{L})$ & 1.5 & 1.6 & 3.8 & 2.2 & & & 2.6 & \\
\hline Adrenaline (nmol/L) & 0.2 & $<0.16$ & 18 & 13 & & & 2.1 & \\
\hline DHEA-s $(\mu \mathrm{mol} / \mathrm{L})$ & 8.4 & 8.1 & 8.6 & 9.3 & & & 1.0 & \\
\hline \multicolumn{9}{|l|}{ Patient 9} \\
\hline Cortisol (nmol/L) & 510 & 490 & 8200 & 16,300 & & & & \\
\hline Aldosterone (pmol/L) & $<30$ & $<30$ & 585 & 1410 & 19.5 & 47.0 & & 1.2 \\
\hline Noradrenaline $(\mathrm{nmol} / \mathrm{L})$ & 0.6 & 0.4 & 5.7 & 4.4 & & & 2.6 & \\
\hline Adrenaline (nmol/L) & $<0.02$ & $<0.02$ & 29 & 22 & & & 2.6 & \\
\hline DHEA-s $(\mu \mathrm{mol} / \mathrm{L})$ & 3.1 & 2.9 & 4.7 & 6.1 & & & 1.5 & \\
\hline
\end{tabular}


Table 2 (continued)

\begin{tabular}{|c|c|c|c|c|c|c|c|c|}
\hline & Peripheral & Vena cava & Left adrenal & Right adrenal & L SI & R SI & $\mathrm{R} / \mathrm{L}$ gradient & $\mathrm{L} / \mathrm{R}$ gradient \\
\hline \multicolumn{9}{|l|}{ Patient 10} \\
\hline Cortisol (nmol/L) & 210 & 230 & 5030 & 3930 & & & & \\
\hline Aldosterone (pmol/L) & 1220 & 302 & 245 & 186 & * & * & 1.0 & \\
\hline Noradrenaline $(\mathrm{nmol} / \mathrm{L})$ & 1.1 & 0.4 & 3.2 & 3.6 & & & & 1.5 \\
\hline Adrenaline (nmol/L) & 0.3 & $<0.16$ & 26 & 55 & & & & 2.7 \\
\hline DHEA-s $(\mu \mathrm{mol} / \mathrm{L})$ & 0.13 & 0.12 & 0.2 & 0.2 & & & & 1.3 \\
\hline \multicolumn{9}{|l|}{ Patient 11} \\
\hline Cortisol (nmol/L) & 280 & 260 & 6240 & 12300 & & & & \\
\hline Aldosterone (pmol/L) & 230 & 254 & 3870 & 16000 & 16.8 & 69.6 & & 2.1 \\
\hline Noradrenaline $(\mathrm{nmol} / \mathrm{L})$ & 0.3 & $<0.18$ & 2.2 & 2.6 & & & 1.7 & \\
\hline Adrenaline (nmol/L) & $<0.16$ & $<0.16$ & 12 & 13 & & & 1.8 & \\
\hline DHEA-s $(\mu \mathrm{mol} / \mathrm{L})$ & 0.9 & 0.8 & 2.1 & 3.0 & & & 1.4 & \\
\hline
\end{tabular}

DHEA-s dehydroepiandrosterone-sulfate, $L$ left, $R$ right, $S I$ selectivity index

*Not calculated due to laboratory error of the peripheral sample

\section{Discussion}

In our cohort, which included patients with ACTHindependent hypercortisolism and bilateral adrenal lesions or normal adrenal glands, AVS contributed to an appropriate choice of unilateral adrenalectomy in two out of ten patients. The study demonstrates that AVS in these cases was valuable, and facilitated avoidance of chronic treatment with steroidogenesis inhibitors or inappropriate bilateral adrenalectomy. Furthermore, two of our patients had normal adrenal glands on CT. In these cases, the AVS was useful for two reasons. Firstly, it confirmed that the excessive cortisol production was of adrenal origin, and secondly, it excluded laterality and thereby supporting the choice of bilateral adrenalectomy.

Unilateral minimally invasive adrenalectomy is the primary treatment of choice for patients with CS caused by unilateral cortisol producing adrenal adenomas. On the other hand, no consensus exists regarding treatment of patients with ACTH-independent $\mathrm{CS}$ and bilateral or undetectable lesions. Diagnosing PPNAD preoperatively can be difficult since other signs of the Carney complex are not always present, not all patients have the otherwise typical PRKAR1A mutation [22], and variations in the appearance of the adrenal glands on CT. In fact, more than half of patients with PPNAD have either morphologically normal adrenal glands, or micronodules that can be difficult to detect, on CT. Similarly, preoperative diagnosis of PBMAH is difficult and can easily be confused with bilateral adrenal adenoma, either functioning or nonfunctioning. Patients with either PPNAD or PBMAH are usually treated by bilateral adrenalectomy, although unilateral adrenalectomy has been reported to be efficacious in some of them $[3,23,24]$. The potential benefit of unilateral adrenalectomy in patients with PBMAH is a preserved adrenal function, while a risk for subsequent recurrence remains. In a retrospective study of 15 patients with PBMAH who underwent unilateral adrenalectomy, where the larger gland according to CT was removed, recurrence was observed in two of fifteen patients after a follow-up of 7 and 8 years respectively [25]. Our patient with PBMAH (Subject 8) who underwent bilateral adrenalectomy had a slightly larger right adrenal gland on a CT scan $(50 \times 47 \mathrm{~mm}$ versus $41 \times 32 \mathrm{~mm}$ ). In some centers, based on the size difference, our patient would have been treated with rightsided adrenalectomy. The AVS, however, with a side-toside cortisol/aldosterone gradient of 1.1, demonstrated a bilateral cortisol production, supporting the choice of bilateral adrenalectomy.

In some centers, adrenocortical scintigraphy with ${ }^{131} \mathrm{I}$ methylnorcholesterol (NP-59) is used to evaluate if adrenal activity in patients with CS is uni- or bilateral $[22,25-27]$. In primary aldosteronism, the sensitivity of this method depends on the size of the adenoma, and is not helpful in cases of small adenomas [28]. Furthermore, since the availability of this method is limited in many countries, AVS could instead be a valuable option to assess whether the cortisol production in these patients is uni- or bilateral. It should however be stressed that AVS is an invasive and technically difficult procedure that should only be performed by an experienced and dedicated interventionist [20].

In patients with primary aldosteronism, cortisol concentrations in the right and left adrenal veins as well as in a peripheral vein are used to evaluate whether the catheterization is successful. A widely accepted criteria for successful catheterization is a central to peripheral gradient $\geq 5$ under ACTH stimulation, i.e. that cortisol concentrations in the adrenal veins should be at least five time higher than the concentration in a peripheral vein, and $\geq 2$ without ACTH stimulation $[9,21]$. How successfulness of the cannulation during AVS is best evaluated in patients with ACTH- 


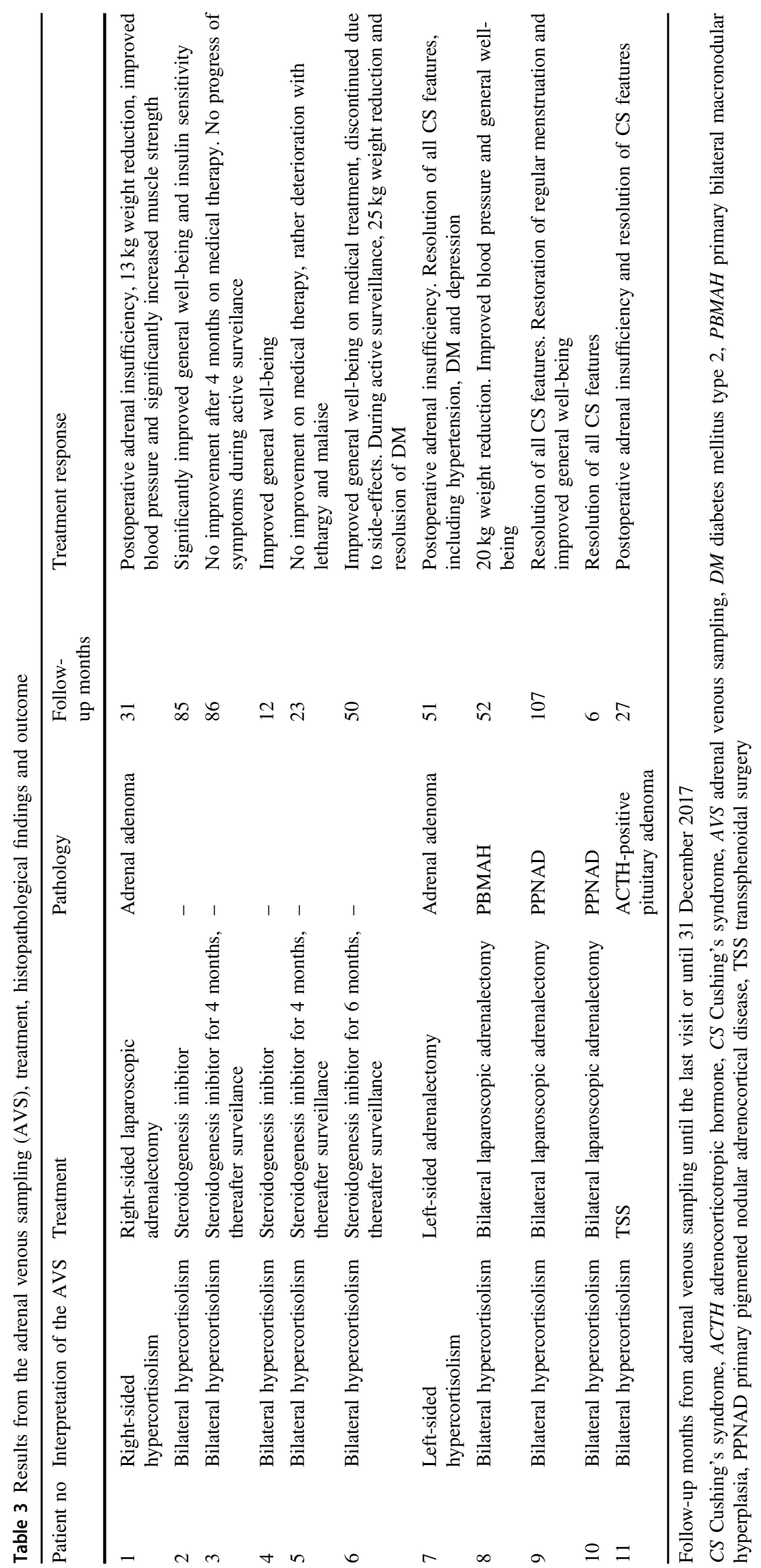


Fig. 1 Patient with mild autonomous cortisol secretion (MACS) and bilateral adrenal lesions (right lesion $32 \times 18 \mathrm{~mm}$ and left lesion $14 \times 8 \mathrm{~mm}$; Subject 1). AVS showed rightsided cortisol overproduction and right-sided adrenalectomy was performed. Postoperatively the patient developed transient adrenal insufficiency
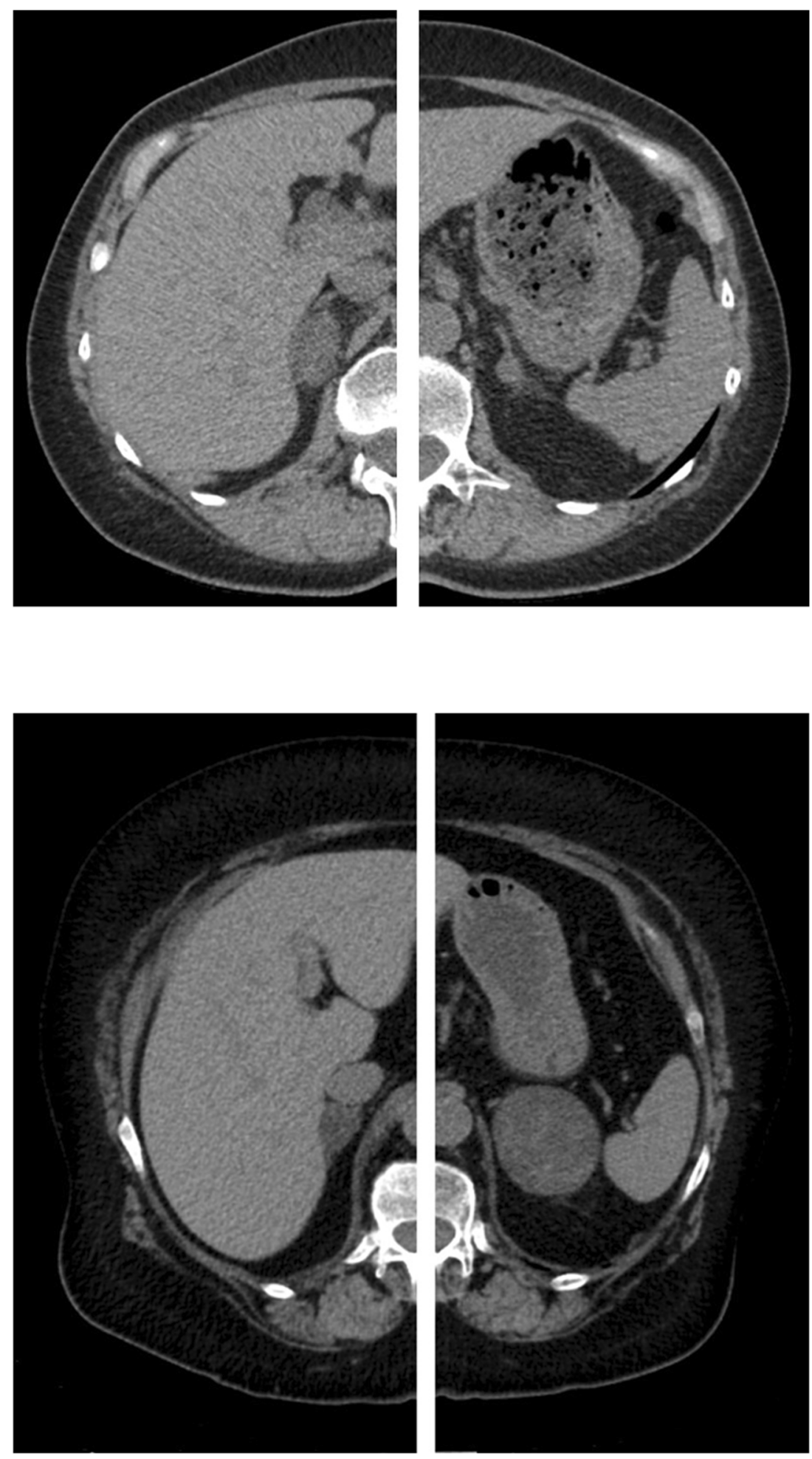

Fig. 2 Patient with overt CS and bilateral adrenal lesions (right lesion $22 \times 15 \mathrm{~mm}$ and left lesion $64 \times 50 \mathrm{~mm}$; Subject 7). AVS showed left-sided cortisol production and the patient underwent left-sided adrenalectomy. Postoperatively the patient developed adrenal deficiency. After 51 months of follow-up the patient has remained in remission independent CS is however not known. The AVS protocols from previous reports varies significantly [8, 10-13, 16-18]. In some cases dexamethasone suppression prior to AVS has been used in order to suppress physiologic endogenous ACTH secretion [8, 10, 16] while in others it has not [13]. AVS has also been performed during simultaneous ACTH stimulation [17]. The interpretation of adequate catheterization varies as well. In one report the radiographic localization of the catheter tip during collection of the adrenal samples was used [12]. In other reports measurements of noradrenaline and adrenaline [13], or only adrenaline $[10,16]$, were used for this purpose. In the report from the 
Fig. 3 Patient with CS and bilateral lesions (right lesion $50 \times 47 \mathrm{~mm}$ and left lesion $41 \times$ $32 \mathrm{~mm}$; Subject 8). AVS showed bilateral cortisol production, and bilateral adrenalectomy was performed. The histopathological diagnosis was PBMAH
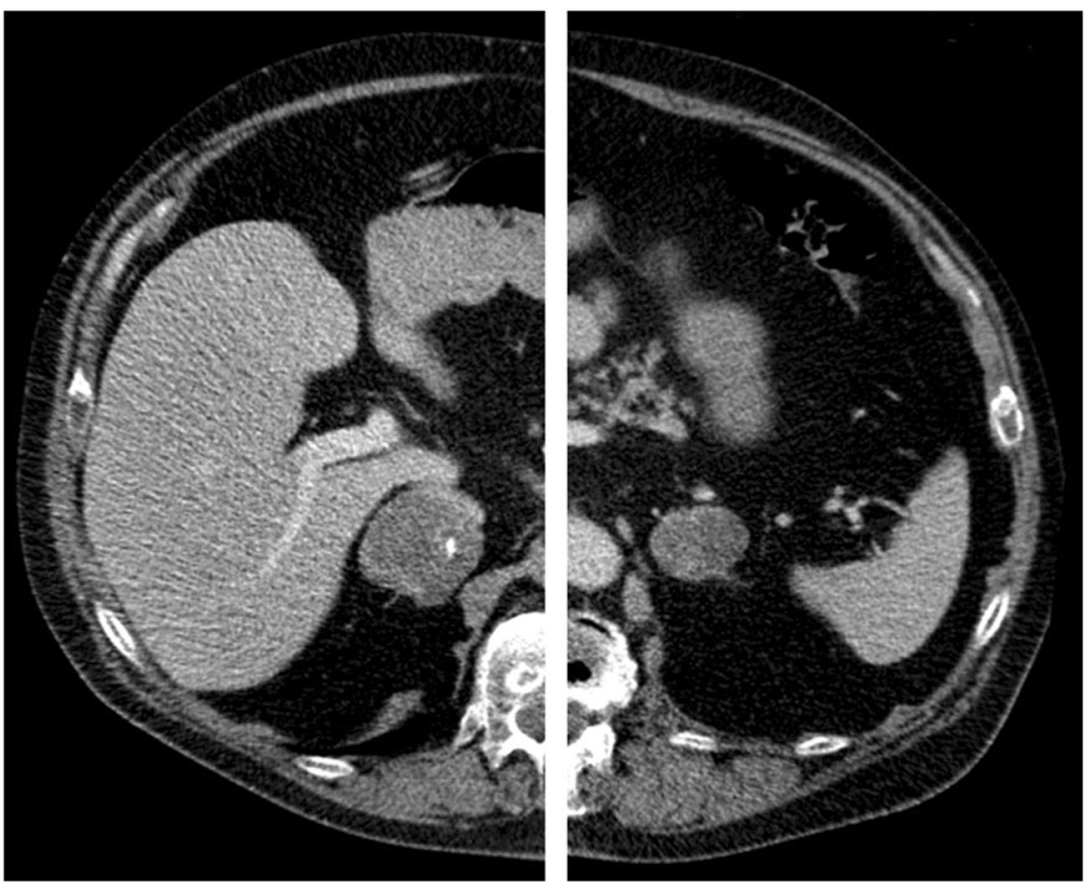

Mayo Clinic, a catheterization was defined as successful if the difference between plasma adrenaline concentration in an adrenal vein was higher than $100 \mathrm{pg} / \mathrm{mL}(0.56 \mathrm{nmol} / \mathrm{L})$ compared to a peripheral vein [10]. In our cohort, adrenaline concentrations were measured in eight out of ten patients. If we had applied the same criteria, one cannulation would have been considered unsuccessful (Subject 7). The usefulness of catecholamines concentrations during AVS has, however, been questioned [29, 30] due to unacceptably wide side-to-side differences, as was observed in three out of nine of our patients (Subjects 3, 5, 7; Table 2), short half-life, as well as large inter-individual variations. Furthermore, catecholamines derive from the adrenal medulla and may therefore not, necessarily, represent the venous drainage from the adrenal cortex. However, in a recent study, plasma metanephrine in the adrenal veins and peripheral vein were used for the assessment of the catheterization. Metanephrine was suggested to be a promising reference hormone for this purpose, both due to its short half-life and that its secretion is not correlated to stress $[8,31]$.

Similarly, the criteria for evaluation of laterality in patients with hypercortisolism have not been established. In the report from the Mayo clinic, including 10 patients with ACTH-independent CS and bilateral adrenal masses [10], an adrenal vein to peripheral vein gradient greater than 6.5 was considered to be consistent with a cortisol-secreting adenoma and a cortisol lateralization ratio (side-to-side adrenal vein cortisol ratio) $\geq 2.3$ consistent with unilateral dominant disease [10]. The same criteria for unilateral disease was used by Ueland et al. [8]. Applying the same criteria for lateralization in our cohort, one patient (Subject 7) would have been misclassified as having bilateral disease (left-to-right cortisol lateralization ratio 1.65). A possible explanation in that case was that the catheterization of the left adrenal vein was semi-selective, and illustrates simultaneously the importance of a collection of a reference hormone(s) in order to be able to take potential diluting effects into account. DHEAS was also collected during the AVS in our study. However, the use of DHEAS as a reference hormone is probably not suitable due to its long half-life $(10 \mathrm{~h})$.

To our knowledge, aldosterone has only been used once previously to correct for possible dilution [12]. Although we found side-to-side cortisol/aldosterone ratio to be useful in the two patients who eventually were treated by unilateral adrenalectomy, the role of measuring aldosterone remains to be further evaluated. The use of aldosterone as a reference hormone in order to correct the dilution differences may have limitations. Stress induced fluctuations in aldosterone secretion, that are minimized by ACTH stimulation during AVS for primary aldosteronism [9], cannot be eliminated during AVS in patients with ACTH-independent CS. Also, aldosterone has a shorter half-life $(20 \mathrm{~min})$ than cortisol (60-70 min) that may interfere in the interpretation of AVS findings. Furthermore, aldosterone and cortisol coproducing adenomas may be more common than previously thought [32], making interpretation of both successfulness as well as laterality difficult. In fact, one patient (Subject 6) in our cohort had a strikingly high aldosterone concentration from the left adrenal vein, due to a concomitant primary aldosteronism. Another patient had 
markedly elevated aldosterone concentrations from the peripheral vein (Subject 10), most probably explained by a laboratory error since repeated measurement of aldosterone and renin was normal.

A major limitation of this study is the small number of patients and the retrospective design. Although the same AVS protocol for the technical procedure was used throughout the study, the interpretation of the AVS results was not predefined, neither the criteria for adrenal vein selectivity nor laterality. Instead, each AVS was evaluated individually. In general, and without any firm scientific support, patients with side-to-side cortisol/aldosterone ratio $>2$ were considered to have unilateral dominant disease, especially when the side-to-side cortisol to adrenaline and noradrenaline, respectively, were also $>2$, with dominance on the same side. Both patients who fulfilled these criteria were operated with unilateral adrenalectomy and developed adrenal insufficiency postoperatively. However, it is important to emphasize that the beneficial effect of bilateral adrenalectomy in patients with overt CS, and apparent bilateral cortisol production according to the AVS, should be interpreted with caution due to lack of independent evidence of unilateral cortisol production with another modality such as adrenal scintigraphy. Another approach in such cases could be unilateral adrenalectomy, where the larger adrenal gland is removed, and the remaining gland would only be removed if the hypercortisolism remained. In fact, if this approach would have been used in subject 1, AVS would not have been necessary. Due to the limited size of the cohort, further studies are however needed before general recommendations concerning criteria for successful catheterization and significant laterality can be provided. Another limitation is that dexamethasone was not measured to assure adequate bioavailability and that more than one assay was used for most of the hormones during the study period. The latter does, however, unlikely affect the main results since these are based on quotients rather than concentrations.

In conclusion, this study on consecutive patients with ACTH-independent hypercortisolism showed that AVS contributed to appropriate unilateral adrenalectomy in two out of ten patients. Knowledge on the usefulness of AVS in patients with ACTH-independent hypercortisolism is still emerging, but the method may, in some cases, be a helpful tool for appropriate therapeutic decision, especially if other modalities are not available.

Acknowledgements Open access funding provided by University of Gothenburg.

\section{Compliance with ethical standards}

Conflict of interest E.P., H.J., A.S., A.M., B.W., and O.E. declare that they have no conflict of interest. G.J. has received lecture fees from Ipsen, NovoNordisk, Otsuka, and Pfizer. O.R. has received lecture fees from Novo Nordisk, Ipsen, Sandoz, and Pfizer, an unrestricted research grant from HRA-pharma, and consultancy fees from Novartis and HRA-pharma.

Ethical approval All procedures performed in studies involving human participants were in accordance with the ethical standards of the Regional Ethical Review Board in Gothenburg, Sweden (reference number 814/18) and with the 1964 Helsinki declaration and its later amendments or comparable ethical standards.

Open Access This article is distributed under the terms of the Creative Commons Attribution 4.0 International License (http://crea tivecommons.org/licenses/by/4.0/), which permits unrestricted use, distribution, and reproduction in any medium, provided you give appropriate credit to the original author(s) and the source, provide a link to the Creative Commons license, and indicate if changes were made.

\section{References}

1. J. Newell-Price, X. Bertagna, A.B. Grossman, L.K. Nieman, Cushing's syndrome. Lancet 367(9522), 1605-1617 (2006)

2. A. Lacroix, R.A. Feelders, C.A. Stratakis, L.K. Nieman, Cushing's syndrome. Lancet 386(9996), 913-927 (2015)

3. A. Lacroix, I. Bourdeau, Bilateral adrenal Cushing's syndrome: macronodular adrenal hyperplasia and primary pigmented nodular adrenocortical disease. Endocrinol. Metab. Clin. North Am. 34(2), 441-458 (2005)

4. I. Chiodini, Clinical review: diagnosis and treatment of subclinical hypercortisolism. J. Clin. Endocrinol. Metab. 96(5), 1223-1236 (2011)

5. G. Osella, M. Terzolo, G. Borretta, G. Magro, A. Ali, A. Piovesan et al. Endocrine evaluation of incidentally discovered adrenal masses (incidentalomas). J. Clin. Endocrinol. Metab. 79(6), 1532-1539 (1994)

6. L. Barzon, C. Scaroni, N. Sonino, F. Fallo, M. Gregianin, C. Macri et al. Incidentally discovered adrenal tumors: endocrine and scintigraphic correlates. J. Clin. Endocrinol. Metab. 83(1), 55-62 (1998)

7. D.A. Vassiliadi, G. Ntali, E. Vicha, S. Tsagarakis, High prevalence of subclinical hypercortisolism in patients with bilateral adrenal incidentalomas: a challenge to management. Clin. Endocrinol. 74(4), 438-444 (2011)

8. G.A. Ueland, P. Methlie, D.E. Jossang, J.V. Sagen, K. Viste, H.B. Thordarson et al. Adrenal venous sampling for assessment of autonomous cortisol secretion. J. Clin. Endocrinol. Metab. 103 (12), 4553-4560 (2018).

9. W.F. Young, A.W. Stanson, G.B. Thompson, C.S. Grant, D.R. Farley, J.A. van Heerden, Role for adrenal venous sampling in primary aldosteronism. Surgery 136(6), 1227-1235 (2004)

10. W.F. Young, Jr. H. du Plessis, G.B. Thompson, C.S. Grant, D.R. Farley, M.L. Richards et al. The clinical conundrum of corticotropin-independent autonomous cortisol secretion in patients with bilateral adrenal masses. World J. Surg. 32(5), 856-862 (2008)

11. S. Leka, E. Kousta, K. Anyfandakis, M. Dolianiti, M. Vakaki, D. Linos et al. Primary pigmented nodular adrenocortical disease: a case report in a 7-year-old girl. J. Pediatr. Endocrinol. Metab. 24 (3-4), 197-202 (2011)

12. C.E. Builes-Montano, C.A. Villa-Franco, A. Roman-Gonzalez, A. Velez-Hoyos, S. Echeverri-Isaza, Adrenal venous sampling in a patient with adrenal Cushing syndrome. Colomb. Med. 46(2), 84-87 (2015)

13. R.G. Martins, R. Agrawal, D.M. Berney, R. Reznek, M. Matson, A.B. Grossman et al. Differential diagnosis of adrenocorticotropic 
hormone-independent Cushing syndrome: role of adrenal venous sampling. Endocr. Pract. 18(6), e153-e157 (2012)

14. E.J. Ku, A.R. Hong, Y.A. Kim, J.H. Bae, M.S. Chang, S.W. Kim, Adrenocorticotropic hormone-independent cushing syndrome with bilateral cortisol-secreting adenomas. Endocrinol. Metab. 28 (2), 133-137 (2013)

15. J.P. Domino, S.B. Chionh, D. Lomanto, A.N. Katara, A. Rauff, W.K. Cheah, Laparoscopic partial adrenalectomy for bilateral cortisol-secreting adenomas. Asian J. Surg. 30(2), 154-157 (2007)

16. Y.W. Guo, C.M. Hwu, J.G. Won, C.H. Chu, L.Y. Lin, A case of adrenal Cushing's syndrome with bilateral adrenal masses. Endocrinol. Diabetes Metab. Case Rep. 2016, 150118 (2016)

17. T. Seki, A. Yasuda, N. Kitajima, M. Oki, A. Takagi, N. Nakamura et al. Adrenal venous sampling is useful for a definitive diagnosis in cushing's syndrome with bilateral adrenal tumors. Tokai J. Exp. Clin. Med. 40(4), 149-156 (2015)

18. A. Maghrabi, A. Yaqub, K.L. Denning, N. Benhamed, S. Faiz, T. Saleem, Challenges in the diagnostic work-up and management of patients with subclinical Cushing's syndrome and bilateral adrenal masses. Endocr. Pract. 19(3), 515-521 (2013)

19. D. Kukidome, N. Miyamura, K. Sakakida, S. Shimoda, Y. Shigematu, K. Nishi et al. A case of cortisol producing adrenal adenoma associated with a latent aldosteronoma: usefulness of the ACTH loading test for the detection of covert aldosteronism in overt Cushing syndrome. Intern. Med. 51(4), 395-400 (2012)

20. H. Jakobsson, K. Farmaki, A. Sakinis, O. Ehn, G. Johannsson, O. Ragnarsson, Adrenal venous sampling: the learning curve of a single interventionalist with 282 consecutive procedures. Diagn. Interv. Radiol. 24(2), 89-93 (2018)

21. J.W. Funder, R.M. Carey, F. Mantero, M.H. Murad, M. Reincke, $\mathrm{H}$. Shibata et al. The management of primary aldosteronism: case detection, diagnosis, and treatment: an endocrine society clinical practice guideline. J. Clin. Endocrinol. Metab. 101(5), 1889-1916 (2016)

22. D. Vezzosi, F. Tenenbaum, L. Cazabat, F. Tissier, M. Bienvenu, C.A. Carrasco et al. Hormonal, radiological, NP-59 Scintigraphy, and Pathological Correlations in Patients With Cushing's Syndrome Due to Primary Pigmented Nodular
Adrenocortical Disease (PPNAD). J. Clin. Endocrinol. Metab. 100(11), 4332-4338 (2015)

23. M. Iacobone, N. Albiger, C. Scaroni, F. Mantero, A. Fassina, G. Viel et al. The role of unilateral adrenalectomy in ACTHindependent macronodular adrenal hyperplasia (AIMAH). World J. Surg. 32(5), 882-889 (2008)

24. R. Guana, R. Gesmundo, M. Morino, P. Matarazzo, A. Pucci, B. Pasini et al. Laparoscopic unilateral adrenalectomy in children for isolated primary pigmented nodular adrenocortical disease (PPNAD): case report and literature review. Eur. J. Pedia. Surg. 20(4), 273-275 (2010)

25. E. Debillon, F.L. Velayoudom-Cephise, S. Salenave, P. Caron, P. Chaffanjon, T. Wagner et al. Unilateral adrenalectomy as a firstline treatment of cushing's syndrome in patients with primary bilateral macronodular adrenal hyperplasia. J. Clin. Endocrinol. Metab. 100(12), 4417-4424 (2015)

26. E.A. Kazerooni, J.C. Sisson, B. Shapiro, M.D. Gross, A. Driedger, G.A. Hurwitz et al. Diagnostic accuracy and pitfalls of [iodine131]6-beta-iodomethyl-19-norcholesterol (NP-59) imaging. J. Nucl. Med. 31(4), 526-534 (1990)

27. A.M. Avram, L.M. Fig, M.D. Gross, Adrenal gland scintigraphy. Semin Nucl. Med. 36(3), 212-227 (2006)

28. G.A. Mansoor, C.D. Malchoff, M.H. Arici, M.K. Karimeddini, G. F. Whalen, Unilateral adrenal hyperplasia causing primary aldosteronism: limitations of I-131 norcholesterol scanning. Am. J. Hypertens. 15(5), 459-464 (2002)

29. Y. Baba, M. Nakajo, S. Hayashi, Adrenal venous catecholamine concentrations in patients with adrenal masses other than pheochromocytoma. Endocrine 43(1), 219-224 (2013)

30. E.M. Freel, A.W. Stanson, G.B. Thompson, C.S. Grant, D.R. Farley, M.L. Richards et al. Adrenal venous sampling for catecholamines: a normal value study. J. Clin. Endocrinol. Metab. 95 (3), 1328-1332 (2010)

31. T. Dekkers, J. Deinum, L.J. Schultzekool, D. Blondin, O. Vonend, A.R. Hermus et al. Plasma metanephrine for assessing the selectivity of adrenal venous sampling. Hypertension 62(6), 1152-1157 (2013)

32. C.R. Lobo, A. Kolinioti, A.J. Hainsworth, G. Bano, S.S. Mudan, A. K. Sharma, Laparoscopic adrenalectomy for co-secreting aldosterone and cortisol adenomas. Int J. Surg. 10(9), 555-559 (2012) 